\title{
LIN28A inhibits lysosome-associated membrane glycoprotein 1 protein expression in embryonic stem and bladder cancer cells
}

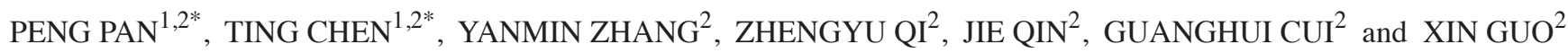 \\ ${ }^{1}$ Department of Pathology, Guangzhou Medical University, Guangzhou, Guangdong 510182; \\ ${ }^{2}$ Guangdong and Shenzhen Key Laboratory of Male Reproductive Medicine and Genetics, \\ Shenzhen, Guangdong 518036, P.R. China
}

Received May 18, 2017; Accepted March 14, 2018

DOI: $10.3892 / \mathrm{mmr} .2018 .8965$

\begin{abstract}
Tumor cells and embryonic stem cells (ESCs) have similar transcription mechanisms. LIN28A is an important factor in tumor cells and ESCs, it is an inhibitor of intracellular endoplasmic reticulum (ER)-related protein translation in ESCs. The present study aimed to examine the effects of LIN28A on an ER-related protein, lysosome-associated membrane glycoprotein 1 (LAMP1), in human bladder cancer cells and mouse (m)ESCs, using reverse transcription-quantitative polymerase chain reaction and western blotting to detect the expression of LAMP1 mRNA and protein, respectively, following LIN28A knockdown. LIN28A was revealed to promote the proliferation, migration and invasion in human bladder cancer cells. These data suggested similarities between ESC cells and cancer cells and may provide novel ideas for the use of induced embryonic stem cell differentiation to treat tumors.
\end{abstract}

\section{Introduction}

LIN28 was first identified in Caenorhabditis elegans as a key control element of embryonic-stage development (1). Mammals have two homologs of LIN28, LIN28A and LIN28B; LIN28B is expressed in the nucleus and regulates the development of embryos by controlling the maturation of LET-7 (2). LIN28A is primarily expressed in embryonic stem cells (ESCs). LIN28A is also able to re-program human fibroblasts into induced pluripotent stem cells, along with octamer-binding protein 4 (OCT4), SOX2 and NANOG, which may indicate a stemness function $(3,4)$. LIN28A may function primarily by inhibiting the maturation of LET-7 microRNA (miRNA) precursors (2).

Correspondence to: Dr Xin Guo, Guangdong and Shenzhen Key Laboratory of Male Reproductive Medicine and Genetics, 1120 Lianhua Road, Shenzhen, Guangdong 518036, P.R. China E-mail: guo_xin73@126.com

*Contributed equally

Key words: LIN28A, lysosome-associated membrane glycoprotein 1 , embryonic stem cells, bladder cancer cells
A number of previous studies have reported that LIN28A is not only expressed in ESCs, but also serves a key role in tumor development (5-9). LIN28A was demonstrated to promote malignant cell transformation and tumor progression, and is also associated with the advanced stages of many tumors, including ovarian carcinoma, hepatocarcinoma, germ cell tumors and lung carcinoma (6-10); however, the role in bladder cancer remains poorly understood.

A previous study (11) have suggested that in addition to targeting LET-7 precursors, LIN28A also targets several other miRNAs and is an inhibitor of endoplasmic reticulum (ER)-related protein translation in ESCs LAMP1 is a membrane glycoprotein and an ER-related protein that functions to maintain the integrity of the lysosomal membrane and serves a role in protein transport, membrane fusion and the transport of intracellular degradation of protein $(12,13)$. However, whether LIN28A affects the translation of LAMP1 proteins in ESCs or in cancer cells is unclear. The process of ESC differentiation is accompanied by a loss of immortality, and the formation of tumor cells indicates that the cells acquire immortality and multi-differentiation (14), which further suggested that a similar functional status may exist between ESCs and tumor cells.

The present study aimed to determine the effects of LIN28A on LAMP1 expression in mouse (m)ESCs and in human bladder cancer cells, and the results demonstrated that LIN28A inhibited LAMP1 protein synthesis in mESCs and in human cancer cells. In addition, it was also demonstrated that LIN28A is able to maintain human bladder cancer cell proliferation migration and invasion.

\section{Materials and methods}

Cell culture. mESCs (National Key Laboratory of Stem Cell and Reproductive Biology, Chinese academy of sciences; Beijing, China) were cultured in mESC media: Dulbecco's modified Eagle medium (DMEM; Thermo Fisher Scientific, Inc., Waltham, MA, USA) containing $15 \%$ fetal bovine serum (FBS; Thermo Fisher Scientific, Inc.), 100 mM Minimum Essential Medium/Non-essential Amino Acids medium (Thermo Fisher Scientific, Inc.), 1,000 U/ml mLIF (Millipore, Darmstadt, Germany) and 50 mM 2-mercaptoethanol (Thermo Fisher Scientific, Inc.). 
Human bladder cancer cell lines 5637, SW780, T24, J82 and UM-UC3 and human cervical cancer cell line HeLa (Type Culture Collection of the Chinese Academy of Sciences, Shanghai, China) were cultured in DMEM with $1 \%$ L-glutamine (Thermo Fisher Scientific, Inc.) supplemented with 10\% FBS (Thermo Fisher Scientific, Inc.). The normal human urothelial cell line SV-HUC-1 (Type Culture Collection of the Chinese Academy of Sciences) was cultured in F12K medium (Thermo Fisher Scientific, Inc.) supplemented with $10 \%$ FBS and $1 \%$ L-glutamine. Cells were cultured in a standard humidified incubator at $37^{\circ} \mathrm{C}$ in a $5 \% \mathrm{CO}_{2}$ atmosphere.

Transfection of siRNA. One day prior to transfection, $3 \times 10^{4}$ cells were plated in $200 \mu \mathrm{l}$ DMEM with $1 \% \mathrm{~L}$-glutamine and $10 \%$ FBS in 48-well plates. After $24 \mathrm{~h}, 0.6 \mu 1$ LAMP1 siRNA (stock solution $20 \mathrm{pmol} / \mu \mathrm{l}$; Shanghai GenePharma Co., Ltd., Shanghai, China) was added to $40 \mu \mathrm{l}$ Opti-MEM I Reduced Serum medium (Invitrogen; Thermo Fisher Scientific, Inc.) to a final concentration of $50 \mathrm{pM}$. siRNA was gently mixed with Lipofectamine 3000 (Invitrogen, CA, USA) and incubated for $20 \mathrm{~min}$ at room temperature. LIN28A-targeted siRNA (mouse) was used to knock down LIN28A in mESCs, and LIN28A-targeted siRNA (human) and LAMP1-targeted (human) siRNA were used to knock down LIN28A and LAMP1 in human bladder cancer cells. siRNA was designed with the following primers: LIN28A-targeted siRNA (human) (5'-GCAUCUGUAAGU GGUUCAATT-3'), LIN28A-targeted siRNA (mouse) (5'-GCA GTGGAGTTCACCTTTAAG-3'), LAMP1-targeted (human) siRNA (5'-GCCACAGUCGGCAAUUCCUACAAGU-3') and negative control (NC)-siRNA (5'-UUCUCCGAACGUGUC ACGUTT-3'). Cells were harvested $24 \mathrm{~h}$ post-transfection. The cells in the wild type (WT) group did not undergo any treatment.

Total RNA extraction and reverse transcription-quantitative polymerase chain reaction $(R T-q P C R)$. Total RNA was extracted from cells when the confluence reached $80 \%$, using TRIzol reagent (Takara Bio, Inc., Otsu, Japan), according to the manufacturer's protocol. Total RNA $(10 \mu \mathrm{g})$ was reverse transcribed to cDNA using the PrimeScript RT Reagent kit with gDNA Eraser (Takara Bio, Inc.), according to the manufacturer's protocol. qPCR was performed using SYBR Premix Ex Taq (TaKaRa Bio, Inc.). In detail, the cDNAs acquired following RT were diluted 10 times, then $2 \mu \mathrm{l}$ was mixed with $5 \mu \mathrm{l}$ AceQ qPCR SYBR Green Master Mix and $50 \mu \mathrm{M}$ primers $(0.15 \mu \mathrm{l}$ each), and distilled deionized water was added to make a total volume of $10 \mu \mathrm{l}$. The thermocycling conditions used were as follows: Initial denaturation at $95^{\circ} \mathrm{C}$ for $10 \mathrm{~min}$; followed by 35 cycles of amplification at $95^{\circ} \mathrm{C}$ for $10 \mathrm{sec}$ and $58^{\circ} \mathrm{C}$ for $30 \mathrm{sec}$. The samples were amplified using the LightCycler ${ }^{\circledR}$ Nano system (Roche Diagnostics, Basel, Switzerland). The primers listed in Table I; experiments were performed in triplicate. mRNA expression levels were normalized to GAPDH and calculated using the $2^{-\Delta \Delta C a}$ method (15).

Gel electrophoresis. A total of $1 \mu \mathrm{g}$ of cDNA obtained from cells was mixed with $2 \mu \mathrm{l}$ of primers (Table I), $10 \mu \mathrm{l}$ of PCR Master Mix (Thermo Fisher Scientific, Inc.) and $8 \mu \mathrm{l}$ of RNase-free water. A Bio-Rad T100 (Bio-Rad Laboratories, Inc., Hercules, CA, USA) thermocycler was used for amplification. Amplification was performed under the following conditions: Initial denaturation at $98^{\circ} \mathrm{C}$ for $3 \mathrm{~min}$; followed by 40 cycles of denaturation at $98^{\circ} \mathrm{C}$ for $30 \mathrm{sec}$, annealing at $60^{\circ} \mathrm{C}$ for $30 \mathrm{sec}$ and extension at $72^{\circ} \mathrm{C}$ for $30 \mathrm{sec}$; and a final extension at $72^{\circ} \mathrm{C}$ for $5 \mathrm{~min}$. Electrophoresis $(85 \mathrm{~V})$ was performed using $1.2 \%$ agarose gels containing ethidium bromide at room temperature for $120 \mathrm{~min}$. Following completion, the gel was placed in an Ulttima 16si detector (Hoefer, Inc., Holliston, MA, USA) in order to visualize the DNA bands by irradiation using ultraviolet light $(254 \mathrm{~nm})$. Densitometric analysis was performed using E-Editor 2.0 software (Thermo Fisher Scientific, Inc.).

Fluorescence immunocytochemistry. For immunofluorescence staining, $3 \times 10^{4}$ cells were placed onto coverslips and fixed with $4 \%$ paraformaldehyde at room temperature for $30 \mathrm{~min}$, permeabilized in $0.1 \%$ Triton for $15 \mathrm{~min}$, blocked by 5\% bovine serum albumin (Thermo Fisher Scientific, Inc.) at $37^{\circ} \mathrm{C}$ for $30 \mathrm{~min}$, incubated with anti-LAMP1 (1:100; cat. no. 21997-1-AP; ProteinTech Group, Inc., Chicago, IL, USA) or anti-LIN28A primary antibodies (1:100; cat. no. ab46020; Abcam, Cambridge, UK) at $37^{\circ} \mathrm{C}$ for $2 \mathrm{~h}$, followed by incubation with an Alexa Fluor 594-conjugated goat anti-rabbit secondary antibody (1:500; A-11012; Invitrogen; Thermo Fisher Scientific, Inc.) at $37^{\circ} \mathrm{C}$ for $1 \mathrm{~h}$. Nuclei were stained with Hoechst 33342 $(1: 2,000)$ at $37^{\circ} \mathrm{C}$ for $5 \mathrm{~min}$. Images were captured with a Zeiss LSM710 fluorescence microscope (Oberkochen, Germany). mESCs were used as a positive control for LIN28A and LAMP1 expression, SV-HUC-1 cells were used as negative control for LAMP1 expression and HeLa cells were used as a negative control for LIN28A expression $(16,17)$.

Western blot analysis. Proteins were extracted from cells when the confluence reached $80 \%$ using radioimmunoprecipitation assay buffer (Beyotime Institute of Biotechnology, Shanghai, China) supplemented with protease inhibitor cocktail (Sigma-Aldrich; Merck KGaA, Darmstadt, Germany). Protein concentrations were determined using the Pierce BCA Protein assay kit (cat. no. 23227; Thermo Fisher Scientific, Inc.). Protein extracts $(50 \mu \mathrm{g})$ were separated by $12 \%$ SDS-PAGE and transferred to polyvinylidene difluoride membranes (EMD Millipore, Billerica, MA, USA). The proteins were then transferred onto PVDF membranes (EMD Millipore). Following blocking with 5\% non-fat milk (blocking buffer) at room temperature for $1 \mathrm{~h}$, the membranes were incubated overnight at $4{ }^{\circ} \mathrm{C}$ with appropriate primary antibodies diluted in blocking buffer as follows: Rabbit monoclonal lysosome-associated membrane glycoprotein 1 (LAMP1; 1:500; cat. no. 9091S; Cell Signaling Technology, Inc., Danvers, MA, USA), mouse monoclonal LIN28A (1:500; cat. no. 11724-1-AP; ProteinTech Group, Inc., Chicago, IL, USA) and mouse monoclonal GAPDH (1:5,000; cat. no. 51332S; Cell Signaling Technology, Inc.). Following washing with PBS with $0.1 \%$ Tween-20 three times, the membranes were incubated at room temperature for $1 \mathrm{~h}$ with anti-mouse (1:2,000; cat. no. sc-2005; Santa Cruz Biotechnology, Inc., Dallas, TX, USA) or anti-rabbit (1:2,000; cat. no. sc-2004; Santa Cruz Biotechnology, Inc.) horseradish peroxidase-conjugated secondary antibodies. Subsequently, the bands of target proteins were visualized using Chemistar ECL Western Blotting Substrate and Tanon-5200 automatic chemiluminescence image analysis system (both Tanon Science \& Technology, Ltd., Shanghai, China). The relative 
Table I. Sequences of primers used for PCR, position of primers on the gene-specific mRNA and expected amplification length of PCR products.

\begin{tabular}{llcc}
\hline Gene & \multicolumn{1}{c}{ Primer $\left(5^{\prime} \rightarrow 3^{\prime}\right)$} & Position $(\mathrm{nt})$ & Size $(\mathrm{bp})$ \\
\hline LAMP1 & F: CAGATGTGTTAGTGGCACCCA & $459-479$ & 84 \\
& R: TTGGAAAGGTACGCCTGGATG & $542-522$ & 134 \\
LIN28A & F: TGCGGGCATCTGTAAGTGG & $120-138$ & 116 \\
& R: GGAACCCTTCCATGTGCAG & $253-235$ & $231-251$ \\
\end{tabular}

F, forward; LAMP1, lysosome-associated membrane glycoprotein 1; PCR, polymerase chain reaction; R, reverse.

intensity of the target bands were quantified by densitometric scanning using Image $\mathbf{J}$ 1.32 j software (National Institutes of Health, Bethesda, MD, USA).

Co-immunoprecipitation (Co-IP). Proteins were extracted from cells when the confluence reached $70-80 \%$ using $100 \mu \mathrm{l}$ RIPA buffer (Beyotime Institute of Biotechnology) supplemented with $1 \%$ protease inhibitor cocktail (Sigma-Aldrich; Merck KGaA). The concentration of protein was determined using a BCA kit according to the aforementioned protocol. Protein extracted from cells was mixed with Protein A agarose (Santa Cruz Biotechnology, Inc.) and the respective antibodies for $8 \mathrm{~h}$ at $4^{\circ} \mathrm{C}$. The antibodies used were: Rabbit monoclonal LAMP1, mouse monoclonal LIN28A and mouse monoclonal as aforementioned. Subsequently, the combined product was incubated with Dynabeads Protein G (Santa Cruz Biotechnology, Inc.) for $1 \mathrm{~h}$ at $4^{\circ} \mathrm{C}$. The bound proteins were analyzed using western blot, as aforementioned.

Transwell invasion assay. A total of $40 \mu 1$ Matrigel $(0.5 \mathrm{mg} / \mathrm{ml}$; Beckman Coulter, Inc., Brea, CA, USA) was spread onto the upper Transwell chamber and incubated for $4 \mathrm{~h}$ at $37^{\circ} \mathrm{C}$. A total of $1 \times 10^{5}$ cells, harvested $24 \mathrm{~h}$ post-siRNA transfection, were seeded in the upper compartment of a Transwell chamber (Corning Inc., Corning, NY, USA) containing OptiMEM I Reduced Serum Medium (Thermo Fisher Scientific, Inc.) and incubated at $37^{\circ} \mathrm{C}$ for $24 \mathrm{~h}$. The cells in the upper membrane were removed via rinsing with PBS three times. The migrated cells underneath the membrane were stained with $0.1 \%$ crystal violet at room temperature for $30 \mathrm{~min}$, and cells were counted from three random fields per membrane under a light microscope (magnification, $\mathrm{x} 20$ ); the average number of cells was calculated from three independent experiments.

Wound-healing assay. A total of $3 \times 10^{5}$ siRNA-transfected cells were cultured in 6-well plates to form a monolayer at $37^{\circ} \mathrm{C}, 5 \% \mathrm{CO}_{2}$ for $24 \mathrm{~h}$. A scratch was made in the cell monolayer and the migration distances were measured at 0 and $24 \mathrm{~h}$; the average migration distance was calculated from three independent experiments.

Cell viability assay. The viability of bladder cancer cells was quantitatively assessed using the Cell Counting kit-8 (CCK-8) assay according to the manufacturer's protocol at $0,24,48$ and
$72 \mathrm{~h}$ post-transfection with siRNA. The cells were incubated in $10 \mu \mathrm{l}$ of CCK-8 solution (Dojindo Molecular Technologies, Inc., Kumamoto, Japan) at $37^{\circ} \mathrm{C}$ for $1 \mathrm{~h}$. The optical density of each well was determined using a microplate reader (Bio-Rad Laboratories, Inc.); the absorbance was measured at $450 \mathrm{~nm}$.

Statistical analysis. To compare data between different groups, one-way analysis of variance was performed followed by Fisher's least significant difference (for equal variances) or Games-Howell (for unequal variances) post hoc test using SPSS 17.0 software (SPSS Inc., IL, USA). P<0.05 was considered to indicate a statistically significant difference.

\section{Results}

Lamp1 and lin28A are expressed in mESCs and human bladder cancer cells. RT-PCR was used to determine if LAMP1 and LIN28A mRNAs were expressed in five human bladder cell lines (T24, UMUC3, J82, 5637 and SW780), one normal human bladder urothelial cell line (SV-HUC-1) and a mESC line. mESCs were used as a positive control for LIN28A and LAMP1 expression, SV-HUC-1 cells were used as negative control for LAMP1 expression and HeLa cells were used as a negative control for LIN28A $(16,17)$. The results demonstrated that LIN28A and LAMP1 were expressed in all types of human bladder cancer cell lines and in mESCs (Fig. 1).

Three bladder cancer cell lines (UM-UC-3,5637 and SW780) were selected for further experimentation. Results from the immunofluorescence expression assay revealed that LAMP1 and LIN28A proteins were expressed in the cytoplasm of the three bladder cancer cell lines, which means LAMP1 and LIN28A are expressed in three malignant bladder cancer cells with different levels of malignancy, but the relationship is not clear (Fig. 2).

LIN28A inhibits LAMP1 protein expression in mESCs. A previous study reported that LIN28A is a major inhibitor of ER-related protein translation in ESCs (9). As an ER-related protein, the expression of LAMP1 in ESCs may be regulated by LIN28A; therefore, LAMP1 protein expression levels were examined, following the successful knockdown of LIN28A mRNA expression in mESCs (Fig. 3A). In mESCs transfected with LIN28A-siRNA the protein expression level of LAMP was demonstrated to be significantly increased by 1.35-fold compared with expression in NC-siRNA transfected 
cells (Fig. 3B). As the effects of LIN28A on its target gene LET7 are at the post-transcriptional level, the expression levels of LAMP1 mRNA were examined. No significant differences were identified in the expression of LAMP1 mRNA in LIN28A-siRNA treated cells compared with WT and NC-siRNA transfected cells (Fig. 3C), which suggested that LIN28A inhibition of LAMP1 protein expression may be independent of LAMP1 mRNA levels in mESCs.

LIN28A inhibits LAMP1 protein translation in human bladder cancer cells. As LIN28A was revealed to affect the protein expression levels of LAMP1 in mESCs, human bladder cancer cells were subsequently used in LIN28A knockdown experiments to determine if a similar process occurred. Following successful transfection with LIN28A-siRNA (Fig. 4A), LAMP1 mRNA expression levels were unaffected compared with NC-siRNA transfected cells (Fig. 4B). In addition, following knockdown of LIN28A, LAMP1 protein expression levels were significantly increased in UM-UC3, 5637 and SW780 cells by $1.59,1.2$ and 1.35 -fold compared with NC-siRNA cells, respectively (Fig. 4C-E). These results indicated that LIN28A may inhibit the translation of LAMP1 protein in ESCs and in human cancer cells.

To further demonstrate the relationship between LIN28A and LAMP1, Co-IP experiments were conducted, which demonstrated that LIN28A protein interacts with LAMP1 protein (Fig. 5). These data indicated that LIN28A may serve a direct role in the regulation of LAMP1 protein expression.

Effects of LIN28A on cell migration, proliferation and invasion of human bladder cancer cells in vitro. To investigate the effects of LIN28A on cancer cells, LIN28A knockdown was conducted in three types of bladder cancer cells; the migration, invasion and proliferative capacities were measured by wound-healing, invasion and CCK- 8 assays, respectively. The data revealed the three types of bladder cancer cells (UM-UC3, 5637, SW780) markedly reduced the rate of cell migration, invasion and proliferation after the knockout of LIN28A in bladder cancer cells. The migration distance exhibited by si-LIN28A cells in the UM-UC3, 5637 and SW780 cell lines was significantly decreased by $0.72,0.66$ and 0.75 fold compared with the $\mathrm{NC}$ group, respectively (Fig. 6). In addition, the invasive ability of si-LIN28A cells in the UM-UC3, 5637 and SW780 cell lines was significantly decreased by $0.75,0.53$ and 0.63 fold compared with the NC group, respectively (Fig. 7). Furthermore, the proliferative ability of si-LIN28A cells in the UM-UC3, 5637 and SW780 cell lines at the $72 \mathrm{~h}$ time interval was significantly suppressed by $0.52,0.56$ and 0.45 fold compared with the NC group, respectively (Fig. 8). The results demonstrated that LIN28A may suppress the migration, invasion and proliferation of bladder cancer cells.

\section{Discussion}

LIN28A was previously reported to promote protein translation in stem cells (14). In addition, LIN28A was also reported to reduce the translation of proteins in the ER and Golgi lumen (11). Crosslinking-immunoprecipitation-sequencing technology was previously used to reveal that LIN28A inhibits the expression of ER-related proteins in ESCs (11). The present

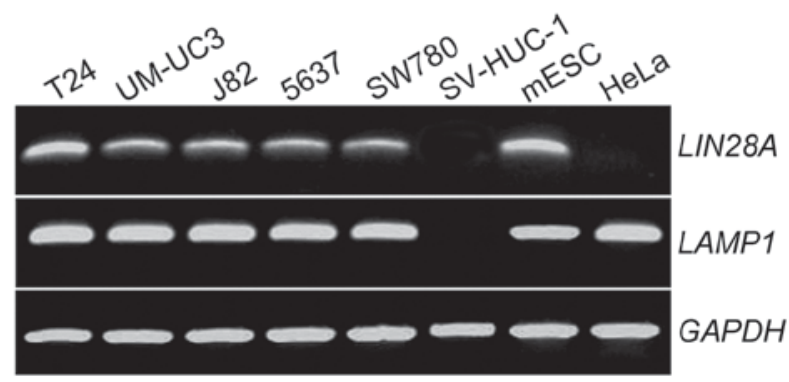

Figure 1. LAMP1 and LIN28A mRNAs are expressed in mESCs and bladder cancer cells. The expression of LAMP1 and LIN28A mRNA was examined by reverse transcription-quantitative polymerase chain reaction in human bladder cancer cell lines T24, UM-UC3, J82, 5637 and SW780, as well as in a normal human urothelial cell line (SV-HUC-1). mESCs were used as a positive control for LIN28A and LAMP1, HeLa cells were used as a negative control for LIN28A expression and SV-HUC-1 cells were used as a negative control for LAMP1. LAMP1, lysosome-associated membrane glycoprotein 1; mESCs, mouse embryonic stem cells

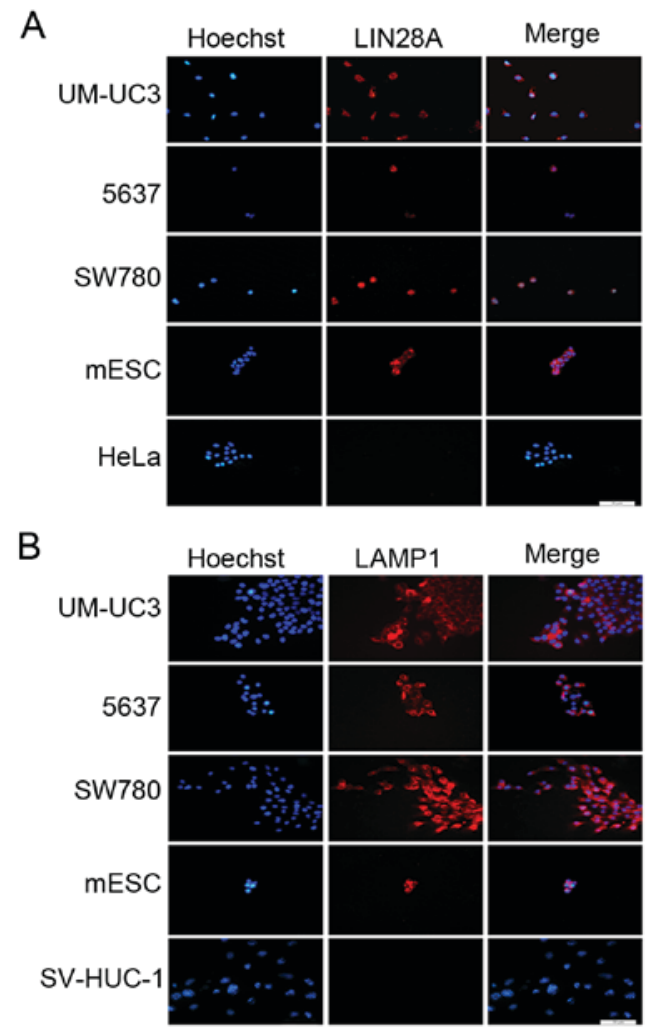

Figure 2. LAMP1 and LIN28A proteins are expressed in human bladder cancer cells and mESCs. Fluorescence immunocytochemical analysis was used to determine the location of (A) LIN28A protein expression in UM-UC3, 5637, SW780, mESCs and HeLa cells and (B) LAMP1 protein expression in UM-UC3, 5637, SW780, mESCs and SV-HUC-1 cells. mESCs were used as a positive control for LIN28A and LAMP1 expression levels, HeLa cells were used as a negative control for LIN28A expression and SV-HUC-1 cells were used as a negative control for LAMP1 expression. Nuclei were stained with Hoechst 33342; scale bar, $50 \mu \mathrm{m}$. LAMP1, lysosome-associated membrane glycoprotein 1; mESCs, mouse embryonic stem cell.

study used western blotting to demonstrate that expression of the ER-related protein LAMP1 was increased following reduced LIN28A expression in mESCs and human bladder cancer cells. Based on these results, it was speculated that the development of ESCs and human tumor cells was comparable. 

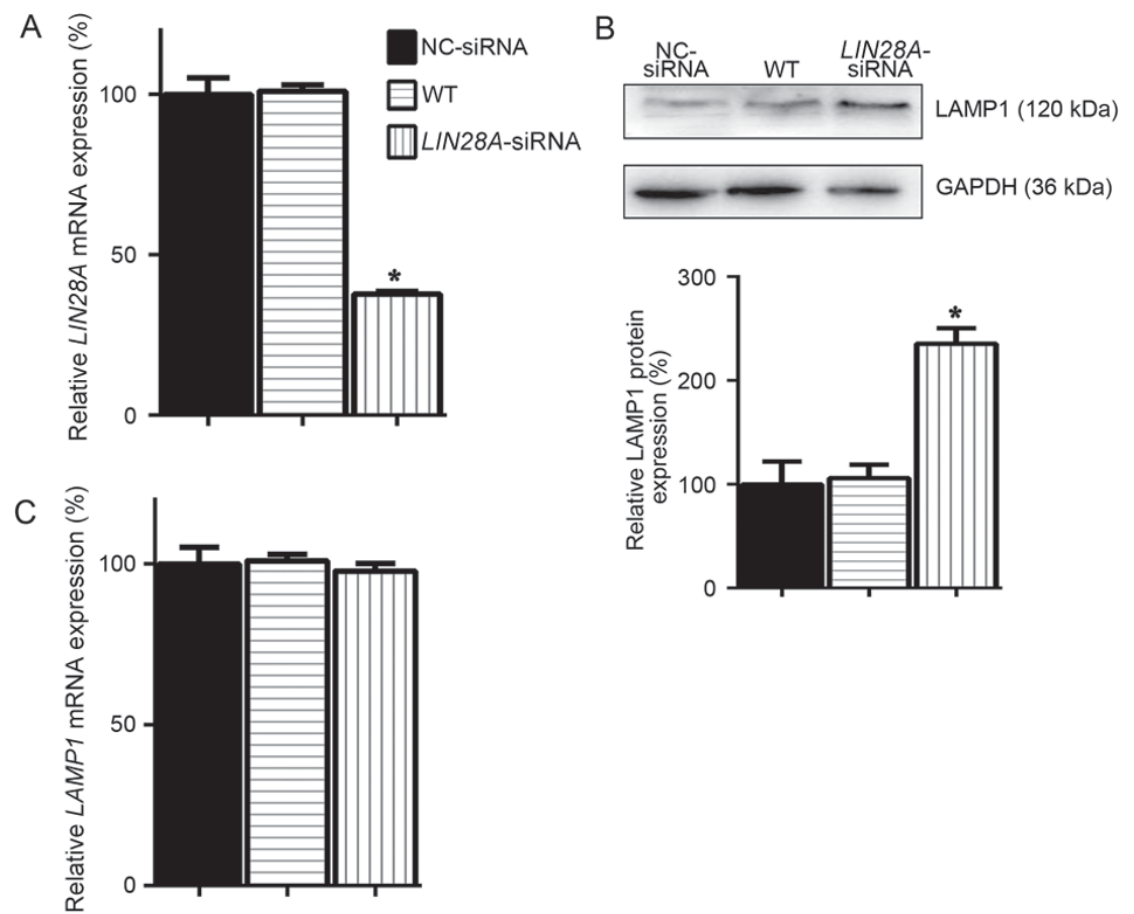

Figure 3. Effects of LIN28A knockdown on LAMP1 protein and mRNA expression levels in mESCs. (A) Results from RT-qPCR demonstrated that LIN28A mRNA expression levels were significantly decreased in mESCs following transfection with LIN28A-siRNA; $\mathrm{n}=3$; ${ }^{*} \mathrm{P}<0.05$ vs. NC-siRNA. (B) mESCs transfected with LIN28A-siRNA exhibited a significant increase in LAMP1 protein expression levels, as determined by western blotting and densitometric analysis; GAPDH was used as a loading control; $\mathrm{n}=3$; ${ }^{*} \mathrm{P}<0.05$ vs. NC-siRNA. (C) No significant differences were identified in the expression levels of lamp1 mRNA in LIN28A-siRNA-transfected cells compared with expression levels in NC-siRNA transfected cells, as determined by RT-qPCR. Data are expressed as the mean \pm standard deviation. Each experiment was performed in triplicate three independent times. LAMP1, lysosome-associated membrane glycoprotein 1; mESCs, mouse embryonic stem cells; NC, negative control; RT-qPCR, reverse transcription-quantitative polymerase chain reaction; siRNA, small interfering RNA; WT, wild-type.
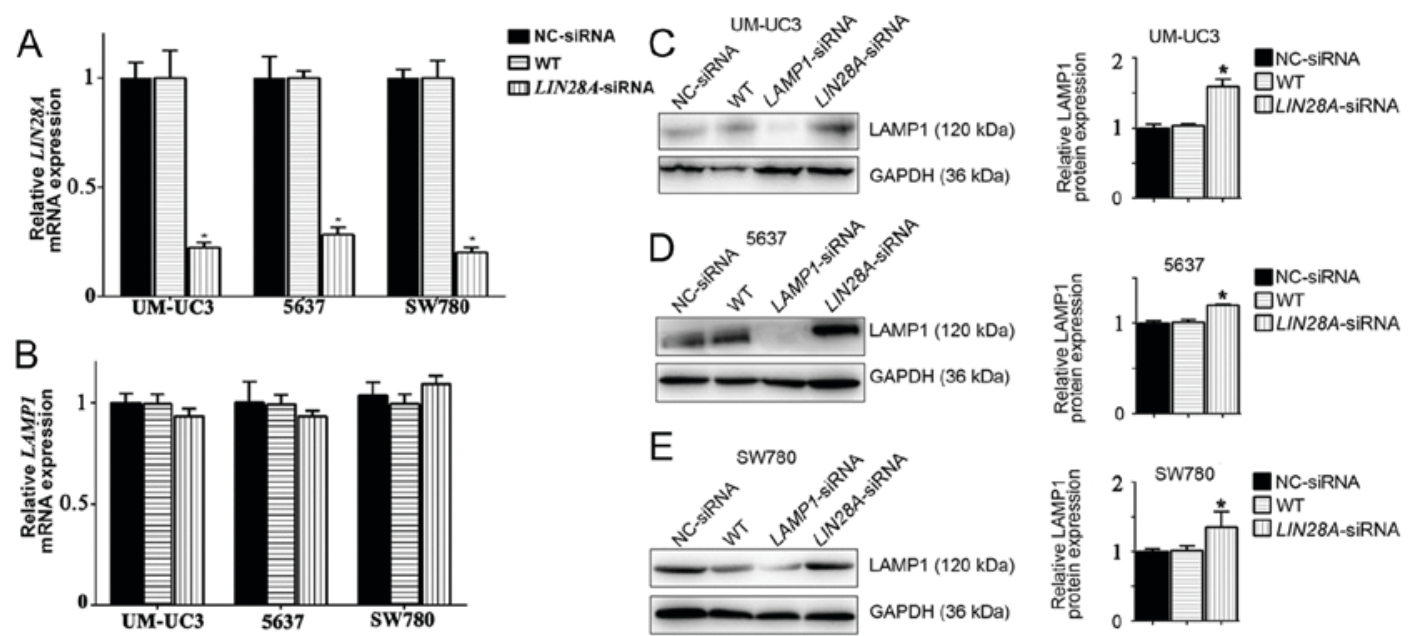

Figure 4. LIN28A knockdown does not affect the expression levels of lamp1 mRNA in human bladder cancer cells while can increase the expression of LAMP1 protein in vitro. (A) Transfection of LIN28A-siRNA in bladder cancer cells (UM-UC3, 5637 and SW780) significantly reduced the mRNA expression levels of LIN28A; $=3$; " $\mathrm{P}<0.05$ vs. NC-siRNA. (B) No significant differences were identified for lamp1 mRNA expression levels in cells transfected with LIN28A-siRNA, compared with NC-siRNA transfected cells. Western blotting analysis demonstrated that the protein expression levels of LAMP1 increased in LIN28A-siRNA transfected (C) UM-UC3, (D) 5637 and (E) SW780 cells. Each experiment was performed in triplicate three independent times. Data are expressed as the mean \pm standard deviation; ${ }^{2} \mathrm{P}<0.05$ vs. NC-siRNA. LAMP1, lysosome-associated membrane glycoprotein 1; NC, negative control; siRNA, small interfering RNA; WT, wild-type.

A number of genes have been previously demonstrated to serve a key role in stem cells, some of which may exhibit oncogene-like functions, such as LIN28A and OCT4 $(8,9)$. In addition, cancer stem cells (CSCs) have been reported to accelerate the process of tumor malignancy (18).
Epithelial-mesenchymal transition has also been reported to serve an important role in ESC differentiation $(19,20)$ and in carcinoma development $(21,22)$.

The present study results suggested that the inhibitory effect of LIN28A on LAMP1 protein expression was at the 


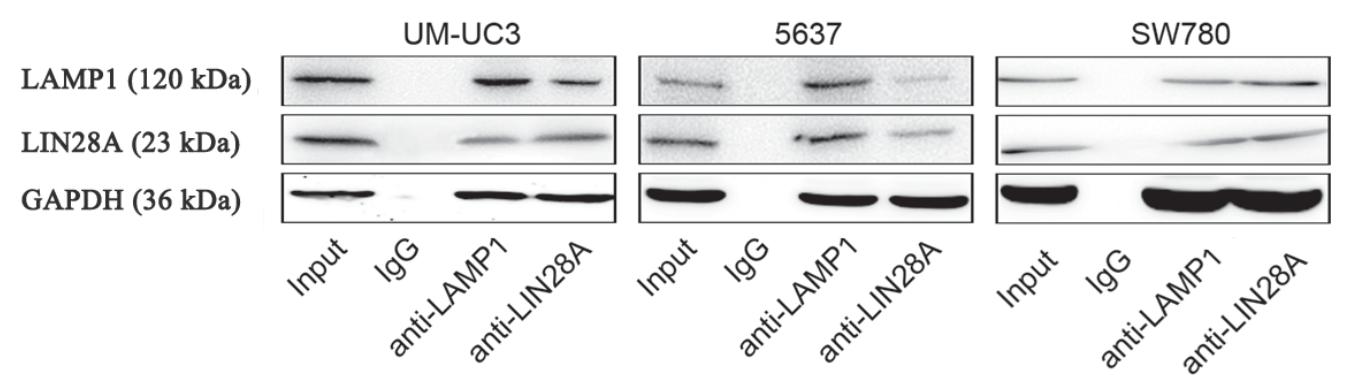

Figure 5. LIN28A interacts with LAMP1 in human bladder cancer cells. Representative western blotting images from co-immunoprecipitation of LAMP1 and LIN28A proteins from UM-UC3, 5637 and SW780 cell lysates using a LAMP1-specific or LIN28A-specific antibody. LAMP1 protein was revealed to co-precipitate with LIN28A. Input protein was used as a positive control; IgG was used as a negative control, GAPDH was used as a loading control. IgG, immunoglobulin G; LAMP1, lysosome-associated membrane glycoprotein 1.

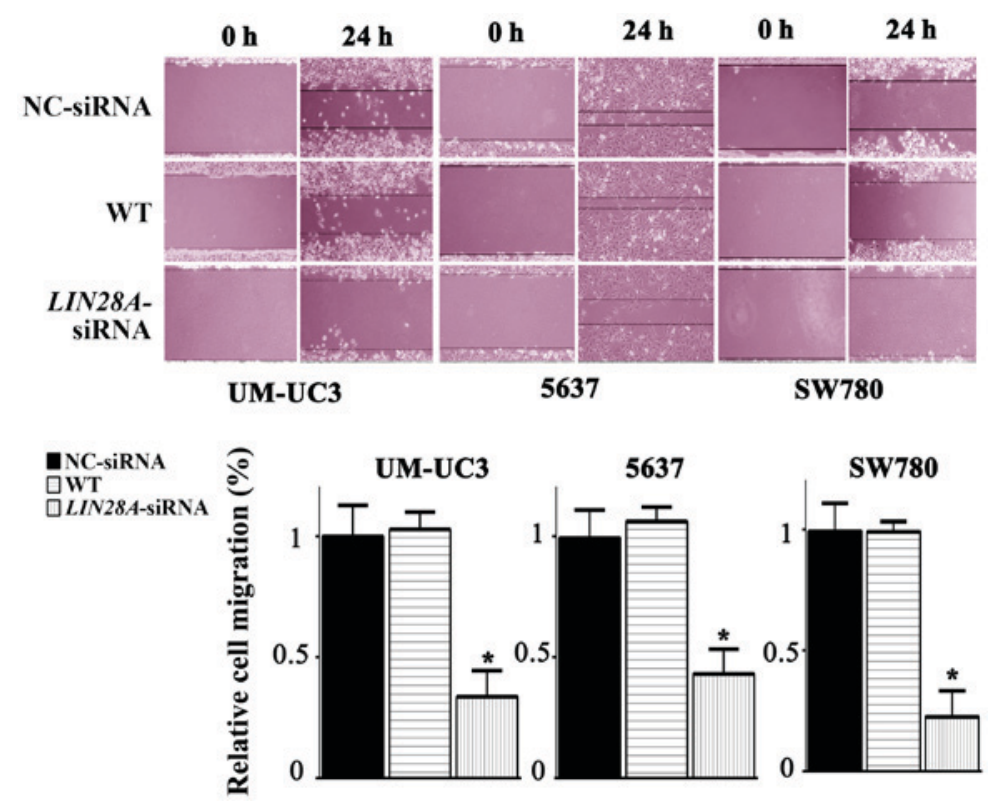

Figure 6. LIN28A knockdown decreases bladder cancer cell motility. Cell motility was measured by wound-healing assay, which demonstrated that LIN28A-siRNA transfection significantly reduced the migratory ability of UM-UC3, 5637 and SW780 cells 24 h post-transfection. Each experiment was performed in triplicate three independent times, and data are presented as the mean \pm standard deviation; ${ }^{2} \mathrm{P}<0.05 \mathrm{vs}$. NC-siRNA. NC, negative control; siRNA, small interfering RNA; WT, wild-type.

post-transcriptional level and did not affect lampl mRNA production in either mESCs or in human bladder cancer cells in vitro. But the expression of LAMP1 protein increased following knockdown of LIN28A, indicating that LIN28A may downregulated the protein expression levels of LAMP1. It has been reported that LIN28A may alter the ribosome structure of some protein synthesis; LIN28A may inhibit the formation of LAMP1 protein without affecting its mRNA expression levels in this way (23). LIN28A was previously reported to post-transcriptionally regulate the expression of let 7 miRNA precursors (24). Recent studies have revealed that, in addition to let7, LIN28A also modifies a number of mRNAs and miRNAs at the post-transcriptional level without affecting RNA synthesis, such as insulin-like growth factor 2 and members of the miRNA-106 family $(25,26)$. These results are consistent with those from the present study.

LIN28A also serves an important role in human tumor cells; an increasing number of studies have reported that the overexpression of LIN28A is one of the biomarkers of CSCs, such as in hepatocellular carcinoma (6), and in ovarian (7), colon (8) and prostate (27) cancers. The present study results demonstrated that LIN28A was expressed in the human bladder cancer cell lines UM-UC3, SW780 and 5637, but not in healthy SV-HUC-1 urothelial cells, and suggested that LIN28A may serve an important role in the development of tumor cells by increasing the proliferation, migration and invasion of bladder cancer cells.

It has been reported that LIN28A affects mitochondrial localization in the cell cytoplasm, which suggested an involvement in oxidative metabolism and oxidative phosphorylation (OXPHOS) (28). Previous studies have also reported that LIN28A is enriched in the intracellular region, including the inner mitochondrial membrane, thus inhibiting cellular oxidative metabolism $(29,30)$. LIN28A was reported to simultaneously repress cell OXPHOS and promote cell anaerobic glycolysis (30). In addition, mitochondrial localization in the cytoplasm is altered following LAMP1 knockdown, which further suggested that LAMP1 may participate in mitochondrial functions (28). Providing the importance of mitochondria in cellular glucose metabolism, LAMP1 may participate in the process of OXPHOS (31). Inhibition of 


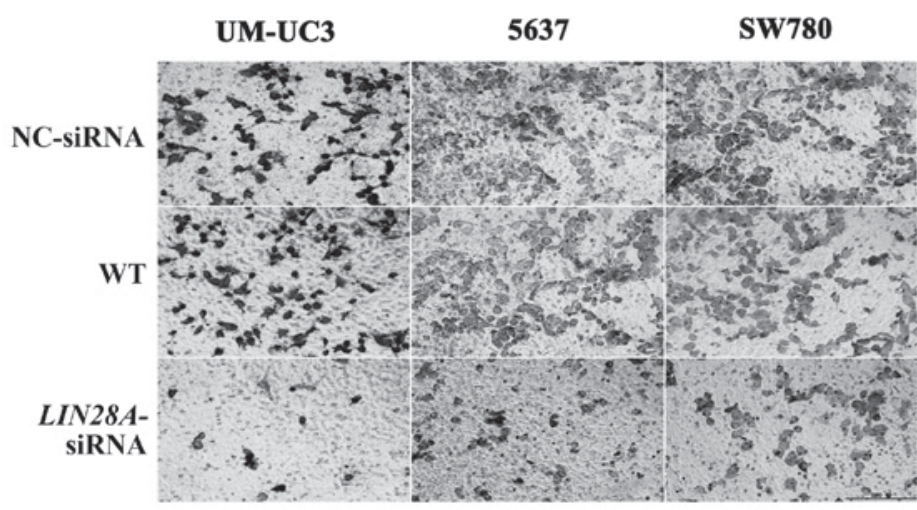

UM-UC3

5637

SW780
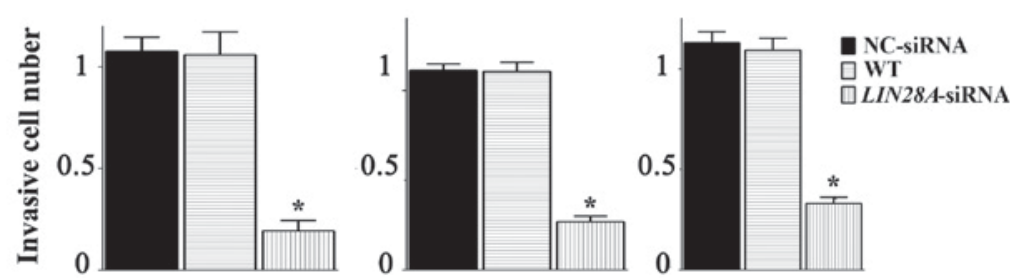

Figure 7. LIN28A knockdown reduces the invasive ability human bladder cancer cells in vitro. Cell invasion was measured by the Transwell invasion assay, which demonstrated that knockdown of LIN28A expression caused a significant decrease in the invasive ability of UM-UC3, 5637 and SW780 cells. Each experiment was performed in triplicate three independent times, and the data are presented as the mean \pm standard deviation; ${ }^{*} \mathrm{P}<0.05 \mathrm{vs}$. WT and NC-siRNA. NC, negative control; siRNA, small interfering RNA; WT, wild-type.
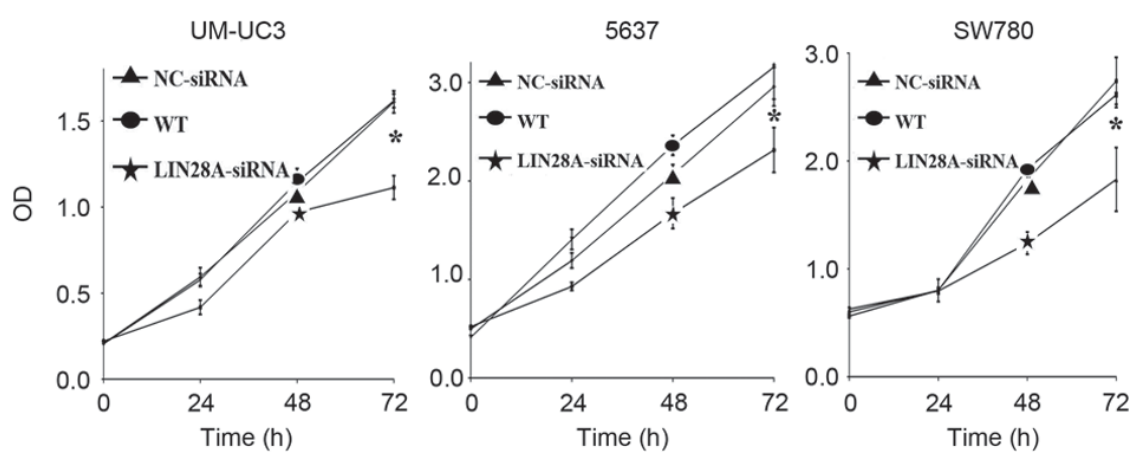

Figure 8. LIN28A knockdown decreases the proliferation of human bladder cancer cells. Cell proliferation was measured with the Cell Counting kit-8 assay at $0,24,48$ and $72 \mathrm{~h}$, which demonstrated a significant decrease in the proliferation of UM-UC3, 5637 and SW780 transfected with LIN28A-siRNA. Each experiment was performed in triplicate three independent times, and the data are presented as the mean \pm standard deviation; ${ }^{2} \mathrm{P}<0.05$ vs. WT and NC-siRNA. NC, negative control; siRNA, small interfering RNA; WT, wild-type.

LAMP1 protein translation may be one of the ways in which LIN28A regulates cell metabolism. LIN28A may inhibit the expression of OXPHOS-associated proteins; LAMP1 is an OXPHOS-associated protein (32). The ER-associated protein LAMP1 is a mitochondria and OXPHOS-related protein that is inhibited by LIN28A.

In conclusion, the results of the present study demonstrated that LIN28A reduced LAMP1 protein expression without inhibiting the level of LAMP1 mRNA expression. The results of the present study, in agreement with Kim et al (33), suggested that embryonic stem cells and tumor cells have similar transcription mechanisms, metabolic pathways and protein synthesis pathways, which may provide a novel treatment approach for the treatment of tumors. Furthermore, the results also suggested that LIN28A may represent a novel target for the treatment of bladder cancer.

\section{Acknowledgements}

We wish to thank Dr Liu for the provision of primer sequences and $\mathrm{Dr}$ Chen for advanced experimental experience of CO-IP. We also wish to thank the staff at MedSci Medicine for the editing of our manuscript.

\section{Funding}

The present study was supported by The National Natural Science Foundation of China (grant no. 81270748).

\section{Availability of data and materials}

The datasets used and/or analyzed during the current study are available from the corresponding author on reasonable request. 


\section{Authors' contributions}

PP, TC, GC and XG made substantial contributions to the conception and design of the study; PP, TC, YZ and ZQ acquired the data; PP, ZQ, JQ, GC and XG performed the analysis and interpretation of data; PP, TC, YZ and JQ drafted and revised the manuscript; and GC and $\mathrm{XG}$ provided the final approval of the version of the manuscript to be published.

\section{Ethics approval and consent to participate}

Not applicable.

\section{Consent for publication}

Not applicable.

\section{Competing interests}

The authors declare that they have no competing interests.

\section{References}

1. Moss EG, Lee RC and Ambros V: The cold shock domain protein LIN-28 controls developmental timing in C. elegans and is regulated by the lin-4 RNA. Cell 88: 637-646, 1997.

2. Piskounova E, Polytarchou C, Thornton JE, LaPierre RJ, Pothoulakis C, Hagan JP, Iliopoulos D and Gregory RI: Lin28A and Lin28B inhibit let-7 microRNA biogenesis by distinct mechanisms. Cell 147: 1066-1079, 2011.

3. Yu J, Vodyanik MA, Smuga-Otto K, Antosiewicz-Bourget J, Frane JL, Tian S, Nie J, Jonsdottir GA, Ruotti V, Stewart R, et al: Induced pluripotent stem cell lines derived from human somatic cells. Science 318: 1917-1920, 2007.

4. Park IH, Zhao R, West JA, Yabuuchi A, Huo H, Ince TA, Lerou PH, Lensch MW and Daley GQ: Reprogramming of human somatic cells to pluripotency with defined factors. Nature 451: 141-146, 2008.

5. Korshunov A, Ryzhova M, Jones DT, Northcott PA, van Sluis P, Volckmann R, Koster J, Versteeg R, Cowdrey C, Perry A, et al: LIN28A immunoreactivity is a potent diagnostic marker of embryonal tumor with multilayered rosettes (ETMR). Acta Neuropathol 124: 875-881, 2012.

6. Tian N, Han Z, Li Z, Zhou M and Fan C: Lin28/let-7/Bcl-xL pathway: The underlying mechanism of drug resistance in Hep3B cells. Oncol Rep 32: 1050-1056, 2014.

7. Enriquez VA, Cleys ER, Da Silveira JC, Spillman MA, Winger QA and Bouma GJ: High LIN28A expressing ovarian cancer cells secrete exosomes that induce invasion and migration in HEK293 Cells. Biomed Res Int 2015: 701390, 2015.

8. Paz EA, LaFleur B and Gerner EW: Polyamines are oncometabolites that regulate the LIN28/let-7 pathway in colorectal cancer cells. Mol Carcinog 1 (53 Suppl): E96-E106, 2014.

9. Murray MJ, Saini HK, Siegler CA, Hanning JE, Barker EM, van Dongen S, Ward DM, Raby KL, Groves IJ, Scarpini CG, et al: LIN28 Expression in malignant germ cell tumors downregulates let-7 and increases oncogene levels. Cancer Res 73: 4872-4884, 2013.

10. Roos M, Rebhan MA, Lucic M, Pavlicek D, Pradere U, Towbin H, Civenni G, Catapano CV and Hall J: Short loop-targeting oligoribonucleotides antagonize Lin 28 and enable pre-let-7 processing and suppression of cell growth in let-7-deficient cancer cells. Nucleic Acids Res 43: e9, 2015.

11. Cho J, Chang H, Kwon SC, Kim B, Kim Y, Choe J, Ha M, Kim YK and Kim VN: LIN28A is a suppressor of ER-associated translation in embryonic stem cells. Cell 151: 765-777, 2012

12. Saftig P and Klumperman J: Lysosome biogenesis and lysosomal membrane proteins: Trafficking meets function. Nat Rev Mol Cell Biol 10: 623-635, 2009.

13. Eskelinen EL: Roles of LAMP-1 and LAMP-2 in lysosome biogenesis and autophagy. Mol Aspects Med 27: 495-502, 2006.
14. Herreros-Villanueva M, Bujanda L, Billadeau DD and Zhang J: embryonic stem cell factors and pancreatic cancer. World J Gastroenterol 20: 2247-2254, 2014

15. Livak KJ and Schmittgen TD: Analysis of relative gene expression data using real-time quantitative PCR and the 2(-Delta Delta C(T)) method. Methods 25: 402-408, 2001.

16. Li MA, Amaral PP, Cheung P, Bergmann JH, Kinoshita M, Kalkan T, Ralser M, Robson S, von Meyenn F, Paramor M, et al: A lncRNA fine tunes the dynamics of a cell state transition involving Lin28, let-7 and de novo DNA methylation. Elife 6: pii: e23468, 2017.

17. Cohnen A, Chiang SC, Stojanovic A, Schmidt H, Claus M, Saftig P, Janßen O, Cerwenka A, Bryceson YT and Watzl C: Surface CD107a/LAMP-1 protects natural killer cells from degranulation-associated damage. Blood 122: 1411-1418, 2013.

18. Chaffer CL, Marjanovic ND, Lee T, Bell G, Kleer CG, Reinhardt F, D'Alessio AC, Young RA and Weinberg RA: Poised chromatin at the ZEB1 promoter enables breast cancer cell plasticity and enhances tumorigenicity. Cell 154: 61-74, 2013.

19. Stryjewska A, Dries R, Pieters T, Verstappen G, Conidi A, Coddens K, Francis A, Umans L, van IJcken WF, Berx G, et al: Zeb2 regulates cell fate at the exit from epiblast state in mouse embryonic stem cells. Stem Cells 35: 611-625, 2017.

20. Cheung LY, Davis SW, Brinkmeier ML, Camper SA and Pérez-Millán MI: Regulation of pituitary stem cells by epithelial to mesenchymal transition events and signaling pathways. Mol Cell Endocrinol 445: 14-26, 2017.

21. Cheng SW, Tsai HW, Lin YJ, Cheng PN, Chang YC, Yen CJ, Huang HP, Chuang YP, Chang TT, Lee CT, et al: Lin28B is an oncofetal circulating cancer stem cell-like marker associated with recurrence of hepatocellular carcinoma. PLoS One 8: e80053, 2013.

22. Yuan WT, Peng CY, Lee SS, Chou MY, Yu CC and Chang YC: Acquisition cancer stemness, mesenchymal transdifferentiation, and chemoresistance properties by chronic exposure of oral epithelial cells to arecoline. Oncotarget 7: 84072-84081, 2016.

23. Shyh-Chang N and Daley GQ: Lin28: Primal regulator of growth and metabolism in stem cells. Cell Stem Cell 12: 395-406, 2013.

24. Seltzer J, Scotton TC, Kang K, Zada G and Carmichael JD: Gene expression in prolactinomas: A systematic review. Pituitary 19: 93-104, 2016.

25. Thakar NY, Ovchinnikov DA, Hastie ML, Gorman J and Wolvetang EJ: RELB alters proliferation of human pluripotent stem cells via IMP3- and LIN28-mediated modulation of the expression of IGF2 and other cell-cycle regulators. Stem Cells Dev 24: 1888-1900, 2015.

26. Warrander F, Faas L, Kovalevskiy O, Peters D, Coles M, Antson AA, Genever P and Isaacs HV: lin 28 proteins promote expression of 17 92 family miRNAs during amphibian development. Dev Dyn 245: 34-46, 2016.

27. Culig Z: Words of wisdom: Re: Lin 28 promotes growth of prostate cancer cells and activates the androgen receptor. Eur Urol 65: 1013, 2014.

28. Rajapakshe AR, Podyma-Inoue KA, Terasawa K, Hasegawa K, Namba T, Kumei Y, Yanagishita M and Hara-Yokoyama M: Lysosome-associated membrane proteins (LAMPs) regulate intracellular positioning of mitochondria in MC3T3-E1 cells. Exp Cell Res 331: 211-222, 2015.

29. Zhu H,Shyh-Chang N, Segrè AV, Shinoda G, Shah SP, Einhorn WS, Takeuchi A, Engreitz JM, Hagan JP, Kharas MG, et al: The Lin28/let-7 axis regulates glucose metabolism. Cell 147: 81-94, 2011.

30. Zhang J, Ratanasirintrawoot S, Chandrasekaran S, Wu Z, Ficarro SB, Yu C, Ross CA, Cacchiarelli D, Xia Q, Seligson M, et al: LIN28 regulates Stem Cell metabolism and conversion to primed pluripotency. Cell Stem Cell 19: 66-80, 2016.

31. Andrzejewski S, Gravel SP, Pollak M and St-Pierre J: Metformin directly acts on mitochondria to alter cellular bioenergetics. Cancer Metab 2: 12, 2014.

32. Docherty CK, Salt IP and Mercer JR: Lin28A induces energetic switching to glycolytic metabolism in human embryonic kidney cells. Stem Cell Res Ther 7: 78, 2016.

33. Kim J, Woo AJ, Chu J, Snow JW, Fujiwara Y, Kim CG, Cantor AB and Orkin SH: A Myc network accounts for similarities between embryonic stem and cancer cell transcription programs. Cell 143: 313-324, 2010. 